That completes the definition. Using the properties of the sequence $\left\langle C_{\alpha}\right| \alpha$ $\left\langle\omega_{2} \& \lim (\alpha)\right\rangle$, it is easy to see that the construction never breaks down, and that for any $\alpha$,

$$
\gamma \in C_{\alpha} \rightarrow(\forall n<\omega)\left(t_{\gamma n}<_{T} t_{\alpha n}\right) .
$$

The proof that $T=\bigcup_{\alpha<\omega_{2}} T_{\alpha}$ is a Souslin $\aleph_{2}$-tree is standard, and $\left\langle\left[\left\langle t_{\alpha n}\right| n\right.\right.$ $\langle\omega\rangle]\left|\alpha<\omega_{2}\right\rangle$ is clearly an $\omega_{2}$-branch of $\boldsymbol{T}^{*}$.

Remark. Using observations of Gregory and others (including ourselves), we see that all that we needed to assume above was $\mathrm{GCH}+\square$. (See [2].)

Theorem 5. Assume V $=\mathrm{L}$. Then there is a Souslin $\aleph_{2}$-tree, $\boldsymbol{T}$, such that $T^{*}$ is Kurepa.

Proof. (Laver) By. $\mathrm{V}=\mathrm{L}$ we can pick some Kurepa $\aleph_{2}$-tree, $K$. We now construct a Souslin $\aleph_{2}$-tree, $T$, much as above. The only difference is that we embed $\boldsymbol{K}$ into $T^{*}$ as we proceed. That is, instead of simply defining one $\omega_{2}$ branch $\left\langle\left[\left\langle t_{\alpha n} \mid n<\omega\right\rangle\right] \mid \alpha<\omega_{2}\right\rangle$ of $\boldsymbol{T}$, we define an entire copy of $\boldsymbol{K}$. The details are easily worked out by comparison with the proof of Theorem 4 , so we shall not go beyond these few remarks.

Remark. The assumption of $\mathrm{V}=\mathrm{L}$ above can be weakened to $\mathrm{GCH}+$ $+\square+$ "there is a Kurepa $\aleph_{2}$-tree."

\title{
References
}

[1] K. J. Devlin, Aspects of constructibility, Springer, Lecture Notes in Math. 354 (1973). [2] - Constructibility, Springer, in preparation.

DEPARTMENT OF MATHEMATICS

UNIVERSITY OF LANCASTER

\section{Topological degree and Sperner's lemma}

by

\section{H. Sies (Hamburg)}

Alstract. Starting with a combinatorial theorem of $\mathbf{K y}$ Fan on pseudomanifolds we define nolom an $n$-dimensional finite polyhedron $/ M$ into $R$ t 2 . In case $I N$ is an $n$-simplex the strong used for finding conditions under which the degree of a map does not vanish. In this way we can generalize some well-known topological results including the fixed point theorems of Brouwer and Kakutani.

Introduction. Sperner's lemma has turned out to be useful in different mathematical fields. Usually this combinatorial result is applied via the celebrated covering theorem for simplexes due to B. Knaster, C. Kuratowski, S. Mazurkiewicz [9]. It is remarkable, however, that the proof of this covering theorem makes use only of the weak form of Sperner's lemma, i.e. only the existence of at least one completely labeled subsimplex is needed, whereas Sperner's lemma states that the number of completely labeled subsimplexes is odd. It is the goal of this paper to improve the mentioned covering theorem by utilizing the strong version of Sperner's lemma and thus to extend classical topological results such as the fixed point theorems of L. E. J. Brouwer and S. Kakutani.

Our approach is closely related to an idea of M. A. Krasnosel'skii [10] who introduced the Brouwer degree $\beta(f$ ) of a continuous map $f$ of an $n$-dimensional closed finite orientable polyhedron into the $n$-dimensional unit sphere on a combinatorial basis. A famous result of $\mathrm{K}$. Borsuk giving a sufficient condition for $\beta(f) \neq 0$ is derived there from a combinatorial antipodal point theorem. In this paper, starting with a combinatorial theorem of Ky Fan [5, Theorem 2] on pseudomanifolds, we shall assign one of the numbers 0,1 to each cnntinuous $\operatorname{map} F: I I^{n} \rightarrow R_{*}^{n+1}$ of an $n$-dimensional finite polyhedron $\Pi^{n}$ (not necessarily closed or orientable) into $R_{*}^{n+1}:=R^{n+1}-\{0\}$ that satisfies a certain boundary condition; this number $\gamma(F)$ will be called degree of $F$ here. For the sake of simplicity we shall do without orientation consideration, so $\gamma$ will be a degree mod 2. Particularly important for the utility of our degree are sufficient conditions for $\gamma(F)=1$. In the most interesting case $\Pi^{n}=\Sigma^{n}$, where $\Sigma^{n}$ denotes the $n$-dimensional unit simplex, such conditions will be obtained from Sperner's lemma. 
§ 1. Preliminaries. Vector inequalities are to be interpreted in the component-wise sense. Throughout this paper let $n$ denote an arbitrary positive integer, $I$ resp. $N$ resp. $R$ the set of integers resp. positive integers resp. real numbers, $(a, b)$ resp. $[a, b]$ the open resp. closed interval for $a, b \in R, R^{n}$ the euclidean $n$-space, $x_{1}, \ldots, x_{n}$ the components of $x \in R^{n}, x y$ the usual scalar product of $x, y \in R^{n}, d$ the metric of $R^{n}$ defined by $d(x, y):=\max \left|x_{i}-y_{i}\right|,\|\cdot\|$ the norm of $R^{n}$ defined by $\|x\|:=\left|x_{1}\right|+\ldots+\left|x_{n}\right|, O^{n-1}:=\left\{x \in R^{n}:{ }^{i}\|x\|=1\right\}$ the unit $(n-1)$-octahedron, $R_{+}^{n}:=\left\{x \in R^{n}: x \geqslant 0\right\}, \Sigma^{n-1}:=O^{n-1} \cap R_{+}^{n}$ the unit $(n-1)$ simplex, $\Sigma_{i}^{n}:=\left\{x \in \Sigma^{n}: x_{i}=0\right\}, R_{*}^{n}:=R^{n}-\{0\}, Q^{n}:=R_{*}^{n}-\left\{x \in R^{n}: x>0\right.$ or $x$ $<0\}, \quad I_{+}(x):=\left\{i: x_{i}>0\right\}, \quad I_{0}(x):=\left\{i: x_{i}=0\right\}, x+\lambda:=x+(\lambda, \lambda, \ldots, \lambda)$ for $x \in R^{n}, \lambda \in R$. For every map $F: S \rightarrow R^{n}\left(S\right.$ a topological space) let $F_{1}, \ldots, F_{n}$ be the components of $F$, and for every real number $\lambda$ let $F+\lambda$ stand for the map of $S$ into $R^{n}$ carrying $x$ into $F(x)+\lambda$; for every homotopy $H: S \times[0,1] \rightarrow R^{n}$ and every $t \in[0,1]$ let $H^{t}: S \rightarrow R^{n}$ be defined by $H^{t}(x):=H(x, t)$.

Simplex resp. complex always means closed euclidean simplex without orientation resp. finite simplicial homogeneously dimensional complex. The topological space $\langle K\rangle$ associated with any $(n-1)$-complex $K$ is called euclidean $(n-1)$-polyhedron. Every topological space homeomorphic to an euclidean $n$ polyhedron is called $n$-polyhedron. To every $n$-complex $K^{n}$ a boundary complex $\partial K^{n}$ is assigned consisting of all faces of those $(n-1)$-simplexes of $K^{n}$ which belong to the faces of exactly an odd number of $n$-simplexes of $K^{n}$. For an $n$ polyhedron $P^{n}$ the boundary and the interior of $P^{n}$ are defined by $\partial P^{n}:=\left\langle\partial K^{n}\right\rangle$ resp. $I\left(P^{n}\right):=P^{n}-\partial P^{n}$, where $K^{n}$ is any complex such that $P^{n}$ and $\left\langle K^{n}\right\rangle$ are homeomorphic.

SPERNER'S LEMMA. Suppose that each vertex $x$ of a simplicial subdivision of the $n$-simplex $\Sigma^{n}$ is labeled by a number contained in $I_{+}(x)$. Then the number of all subsimplexes whose vertices are labeled by $1, \ldots, n+1$ is odd.

Proof. See Sperner [12].

The next two lemmas will later turn out to be useful.

Lemma 1.1. Let $f: \bar{D} \rightarrow O^{n}$ be a continuous injective map of the closed hull of a bounded region $D \subset R^{n}$ into the n-octahedron $O^{n}$. If $A$ is a closed connected subset of $O^{n}$ such that $A \cap f(D) \neq \varnothing$ but $A \cap f(\bar{D}-D)=\varnothing$, then $A \subset f(D)$.

Proof. $f: \bar{D} \rightarrow f(\bar{D})$ is a homeomorphism, hence $f(D)$ is open in $O^{n}$ because of the invariance of region. $f(\bar{D})$ is compact and hence closed in $O^{n}$. Therefore the nonempty set $A \cap f(D)=A \cap f(\bar{D})$ is open and closed in $A$. Since $A$ is connected we have $A \subset f(D)$.

LEMMA 1.2. Let $C^{n}$ be a closed n-cell, i.e. a space homeomorphic to the closed unit $n$-ball $B^{n}$, and let $c \in C^{n}$ be arbitrary. Then $\partial C^{n}-\{c\}$ is a strong deformation retract of $C^{n}-\{c\}$.

Proof. Evidently the assertion is true for $C^{n}=B^{n}$, and hence for any $n$-cell $C^{n}$
Starting point for the introduction of our topological degree is a combinatorial result of Fan [5, Theorem 2]. Its formulation requires some further terminology.

For any complex $K$ let $\hat{K}$ denote the set of vertices of $K$; a map $\varphi$ of $\hat{K}$ into $I$ is called labeling of $K$; for any $n$ distinct integers $j_{1}, \ldots, j_{n}$ let $\alpha_{K}\left(j_{1}, \ldots, j_{n}\right)$ denote the number of all $(n-1)$-simplexes of $K$ whose vertices carry the $\varphi$-labels $j_{1}, \ldots, j_{n}$; if necessary we shall more precisely write $\alpha_{K}^{\varphi}\left(j_{1}, \ldots, j_{n}\right)$.

A labeling $\varphi$ of an $n$-complex $K^{n}$ is called admissible, if it has the föllowing properties:

(1) $0<|\varphi(x)| \leqslant n+1, \forall x \in \hat{K}^{n}$

(2) $\quad \varphi(x) \neq-\varphi(y)$ for the vertices $x, y$ of each 1 -simplex of $K^{n}$;

(3) $\varphi(x)<0, \forall x \in \hat{\partial K^{n}}$.

We are now in a position to state the announced combinatorial result.

Lemma 1.3. Let . $p$ be an admissible labeling of an n-complex $K^{n}$. Then for every combination $\left(\varepsilon_{1}, \ldots, \varepsilon_{n+1}\right)$ of the signs $\varepsilon_{i}= \pm 1$ with at least one $\varepsilon_{i}=1$, the congruence

$\alpha_{K^{n}}\left(\varepsilon_{1} 1, \ldots, \varepsilon_{n+1}(n+1)\right) \equiv \alpha_{K^{n}}(-1, \ldots,-(n+1))+\alpha_{a K^{n}}(-1, \ldots,-n) \bmod 2$ holds.

Proof. See [5] or [11]. .

\& 2. A combinatorial degree for admissible labelings of complexes. Lemma 1.3 says that, under the conditions of that lemma, either all the numbers $\alpha_{K^{n}}\left(\varepsilon_{1} 1, \ldots, \varepsilon_{n+1}(n+1)\right)\left(\varepsilon_{i}= \pm 1\right.$ with at least one $\left.\varepsilon_{i}=1\right)$ are odd or all of them are even. This fact makes it possible to assign to every admissible labeling $\varphi$ of an $n$-complex $K^{n}$ a combinatorial degree $\Gamma(\varphi)$ by the following definition:

$\Gamma(\varphi):=j \in\{0,1\}$, if $\alpha_{K^{n}}\left(\varepsilon_{1} 1, \ldots, \varepsilon_{n+1}(n+1)\right) \equiv j \bmod 2$,

$$
\forall \varepsilon_{i} \in\{-1,1\} \text { with at least one } \varepsilon_{i}=1 \text {. }
$$

The next lemma states that $\Gamma$ is invariant under simplicial subdivision if the extension of the labeling satisfies a certain condition.

LeMma 2.1. Let $\varphi$ be an admissible labeling of an $n$-complex $K$; let $\bar{K}$ be $a$ simplicial subdivision of $K$, and let $\bar{\varphi}: \hat{\bar{K}} \rightarrow I$ be an extension of $\varphi$ with the following property:

(4) $\varphi(\hat{\sigma})=\bar{\varphi}(\hat{\bar{\sigma}})$ for every simplex $\sigma \in K$, where $\bar{\sigma}$ denotes the simplicial subdivision induced on $\sigma$ by $\bar{K}$.

Then $\bar{\varphi}$ is admissible, and $\Gamma(\varphi)=\Gamma(\bar{\varphi})$.

Proof. Obviously $\bar{\varphi}$ is admissible. Every $n$-simplex $\bar{\sigma}$ of $\bar{K}$ whose vertices carry the $\bar{\rho}$-labels $1, \ldots, n+1$ is a subsimplex of an $n$-simplex $\sigma$ of $K$ whose vertices carry the $\varphi$-labels $1, \ldots, n+1$ because of (4). Furthermore, by Sperner's 
lemma such a simplex $\sigma$ contains an odd number of subsimplexes whose vertices carry the $\bar{\varphi}$-labels $1, \ldots, n+1$. These two facts yield $\alpha_{K}^{p}(1, \ldots, n+1)$ $\equiv \alpha_{K}^{\bar{\Phi}}(1, \ldots, n+1) \bmod 2$ and thus $\Gamma(\varphi)=\Gamma(\bar{\varphi})$.

LeMma 2.2. Let $\varphi, \varphi^{\prime}$ be admissible labelings of an $n$-complex $K$. If there is a vertex $v$ of $K$ such that $\varphi(x)=\varphi^{\prime}(x), \forall x \in \hat{K}-\{v\}$, but $|\varphi(v)| \neq\left|\varphi^{\prime}(v)\right|$, then $\Gamma(\varphi)$ $=\Gamma\left(\varphi^{\prime}\right)$.

Proof. Let $i_{1}, \ldots, i_{n}$ be positive integers such that $\left\{i_{1}, \ldots, i_{n}\right\}=\{1, \ldots, n+$ $+1\}-\{|\varphi(v)|\}$. Then

$$
\Gamma(\varphi)=\alpha_{\mathrm{K}}^{\varphi}\left(i_{1}, \ldots, i_{n},-\varphi(v)\right)=\alpha_{\mathrm{K}}^{\varphi^{\prime}}\left(i_{1}, \ldots, i_{n},-\varphi(v)\right)=\Gamma\left(\varphi^{\prime}\right) .
$$

§ 3. Definition of a topological degree $\gamma$. In what follows let $I I^{n}$ resp. $P^{n}$ always denote an arbitrary euclidean $n$-polyhedron resp. an arbitrary npolyhedron; without loss of generality $\Pi^{n}$ will be assumed to be embedded in a euclidean space of sufficiently high dimension.

A map $F: P^{n} \rightarrow R^{n+1}$ is called positive ( $\partial$-negative) if for each $x \in P^{n}$ $\left(x \in \partial P^{n}\right) F(x)$ has at least one positive (negative) component. Analogously we define a $\partial$-nonpositive map.

Let $F: \Pi^{n} \rightarrow R_{*}^{n+1}$ be continuous and $\partial$-negative. Because of the compactness of $\Pi^{n}$ and $\partial \Pi^{n}$ there exists an $\varepsilon>0$ such that $d\left(0, F\left(\Pi^{n}\right)\right)>\varepsilon$ and $d\left(R_{+}^{n+1}, F\left(\partial \Pi^{n}\right)\right)>\varepsilon$. For $\varepsilon$ there is $\delta>0$ such that $x, y \in \Pi^{n}, d(x, y)<\delta$ always imply $d(F(x), F(y))<\varepsilon$. Let $K$ be a simplicial decomposition of $I^{n}$ with mesh $<\delta$ with respect to $d$. Then there is an admissible labeling $\varphi_{F}$ of $K$ with the following property:

(5) If $\varphi_{F}$ assigns the number $j$ to a vertex $x$ of $K$, then the component $F_{|j|}$ of $F$ has the same sign as $j$ on the whole star of $x$, i.e. on all simplexes of $K$ with the vertex $x$

Thus, calling every labeling $\varphi_{F}$ of a simplicial decomposition $K$ of $\Pi^{n}$ which is admissible and satisfies (5) generated by $F$, we have proved:

Lemma 3.1. Every continuous $\partial$-negative map $F: \Pi^{n} \rightarrow R_{*}^{n+1}$ generates a labeling $\varphi_{F}$

LEMMA 3.2. If $\varphi_{1}, \varphi_{2}$ are labelings generated by a continuous $\partial$-negative map $F: \Pi^{n} \rightarrow R_{*}^{n+1}$, then $\Gamma\left(\varphi_{1}\right)=\Gamma\left(\varphi_{2}\right)$.

Proof. If $\varphi_{1}, \varphi_{2}$ are labelings of the same simplicial decomposition $K$ of $\Pi^{n}$, then the assertion is proved by successive application of Lemma 2.2. If $\varphi_{1}$, $\varphi_{2}$ are labelings of different decompositions $K_{1}$ resp. $K_{2}$ of $I I^{n}$, then let $K$ be a common refinement of $K_{1}, K_{2}$. For $i=1,2$ let $\bar{\varphi}_{i}$ be a labeling of $K$ which extends $\varphi_{i}$ in such a way that it assigns to each vertex $x$ of $K$ the $\varphi_{i}$-label of a vertex of the carrier of $x$ in $K_{i}$ (i.e. the simplex of least dimension in $K_{i}$ which includes $x$ ); $\bar{\varphi}_{1}, \bar{\varphi}_{2}$ are labelings of $K$ generated by $F$. From Lemma 2.1 and the first part of this proof we conclude

$$
\Gamma\left(\varphi_{1}\right)=\Gamma\left(\bar{\varphi}_{1}\right)=\Gamma\left(\bar{\varphi}_{2}\right)=\Gamma\left(\varphi_{2}\right)
$$

If $H: P^{n} \times[0,1] \rightarrow R_{*}^{n+1}$ is continuous and if $H^{t}$ is $\partial$-nonpositive for every $t \in[0,1]$, then $H$ is called $\partial$-nonpositive homotopy, and $H^{0}, H^{1}$ are said to be $\partial$ nonpositively homotopic. In an analogous manner we define a $\partial$-negative homotopy.

LeMma 3.3. If $H: I I^{n} \times[0,1] \rightarrow R_{*}^{n+1}$ is a d-negative homotopy, then $\Gamma\left(\varphi_{H^{0}}\right)$ $=\Gamma\left(\varphi_{H^{1}}\right)$.

Pro of. If $K$ is a sufficiently fine simplicial decomposition of $\Pi^{n}$ and $\delta>0$ is sufficiently small, there exists, for all $s, t \in[0,1]$ such that $|s-t|<\delta$, a labeling of $K$ generated both by $H^{s}$ and by $H^{t}$. From this we can easily derive the assertion.

Let $F: \Pi^{n} \rightarrow R_{*}^{n+1}$ be continuous and $\partial$-nonpositive. There is a positive real number $\delta_{F}$ such that $(F-\varepsilon)(x) \neq 0, \forall \varepsilon \in\left(0, \delta_{F}\right), \forall x \in \Pi^{n}$. The maps $F-\varepsilon$ $\varepsilon \in\left(0, \delta_{F}\right)$, are continuous and pairwise $\partial$-negatively homotopic, and hence all labelings $\varphi_{F-B}$ generated by them have the same combinatorial degree. This suggests the following

Definition 3.4. If $F: \Pi^{n} \rightarrow R_{*}^{n+1}$ is continuous and $\partial$-nonpositive, we put

$$
\gamma(F):=\Gamma\left(\varphi_{F-\varepsilon}\right), \quad \varepsilon \in\left(0, \delta_{F}\right) ;
$$

$\gamma(F)$ is called degree of $F$

$\$ 4$. Properties of the degree $\gamma$. Lemma 3.3 can evidently be extended in the following way:

TheOREM 4.1. If $H: \Pi^{n} \times[0,1] \rightarrow R_{*}^{n+1}$ is a $\partial$-nonpositive homotopy, then

$$
\gamma\left(H^{0}\right)=\gamma\left(H^{1}\right) .
$$

Let $F, G$ be maps of $P^{n}$ into $R^{n+1}$. We say that $F, G$ are together $\partial$ monpositive if all maps $s F+t G\left(s, t \in R_{+}\right)$are $\partial$-nonpositive. If there are $\lambda \in R$ $a \in P^{n}$ such that $\lambda F(a)=G^{*}(a)$, then $\lambda$ is called equilibrium value of $(F, G)$; if $P^{n}$ $=\Sigma^{n}$ and if $F$ is the inclusion map defined by $F(x):=x$, then $\lambda$ is called eigenvalue of $\cdot G$.

TheOREM 4.2. Let $F: \Pi^{n} \rightarrow R_{*}^{n+1}, G: \Pi^{n} \rightarrow R^{n+1}$ be continuous and together $\partial$-nonpositive. If $\varrho$ is any nonnegative real number such that $(F, G)$ has no equilibrium value $\leqslant-\varrho$, then $\gamma(F)=1$ implies

$$
\gamma(\varrho F+G)=1 .
$$

Pro of. Suppose $\gamma(F)=1$ and let $\varrho \in R_{+}$be such that (6) is false. Then either $\gamma(\varrho F+G)=0$ or $0 \in(\varrho F+G)\left(\Pi^{n}\right)$. Hence there are $x \in \Pi^{n}, \lambda \leqslant 0$ such that $\lambda F(x)$ $=(\varrho F+G)(x)$, and this means that $(F, G)$ has an equilibrium value $\leqslant-\varrho$.

THEOREM 4.3. Let $f: \Pi^{n} \rightarrow O^{n}$ be a continuous and 0 -nonpositive map such that $\gamma(f)=1$. Then:

(a) $\Sigma^{n} \subset f\left(\Pi^{n}\right)$; 
(b) $\mathscr{C}\left(I\left(\Sigma^{n}\right)\right) \subset f\left(I\left(\Pi^{n}\right)\right)$, where $\mathscr{C}\left(I\left(\Sigma^{n}\right)\right)$ denotes the connected component of $I\left(\Sigma^{n}\right)$ in $O^{n}-f\left(\partial \Pi^{n}\right)$;

(c) $f\left(I\left(\Pi^{n}\right)\right)$ is a neighborhood of $\Sigma^{n}-f\left(\partial \Pi^{n}\right)$ in $O^{n}$.

Pro of. (a) If $g^{a}: I^{n} \rightarrow O^{n}$ denotes the constant map defined by $g^{a}(x):=a$, then $\gamma\left(g^{a}\right)=0, \forall a \in-\Sigma^{n}$. Application of Theorem 4.2 with $\varrho=0, F=f, G=g^{a}$ yields $\Sigma^{n} \subset f\left(\Pi^{n}\right)$.

(b) Let $\mathscr{G}$ denote the set of all continuous $\partial$-nonpositive maps $g: \Pi^{n} \rightarrow O^{n}$ such that $\gamma(g)=1$ and $g\left(\partial \Pi^{n}\right)=f\left(\partial \Pi^{n}\right)$. Consider the set $M:=\left(\bigcap_{g} g\left(\Pi^{n}\right)\right)$ $-f\left(\partial \Pi^{n}\right)$. Part (a) of this theorem yields $I\left(\Sigma^{n}\right) \subset M$. Now we show that $M$ is open and closed in $O^{n}-f\left(\partial \Pi^{n}\right)$. If $p$ is any point of $M$, there is a closed $n$-cell $C \subset O^{n}-f\left(\partial \Pi^{n}\right)$ such that $p \in I(C)$. If $g: \Pi^{n} \rightarrow O^{n}$ is a continuous $\partial$-nonpositive map such that $g\left(\partial \Pi^{n}\right)=f\left(\partial \Pi^{n}\right)$ and $I(C) \notin g\left(\Pi^{n}\right)-g\left(\partial \Pi^{n}\right)$, then, by Lemma 1.2, there is a map $g^{\prime}: \Pi^{n} \rightarrow O^{n}$ such that $g^{\prime}\left(\partial \Pi^{n}\right)=f\left(\partial \Pi^{n}\right)$ and $p \notin g^{\prime}\left(\Pi^{n}\right)-g^{\prime}\left(\partial \Pi^{n}\right)$ and such that $g$ and $g^{\prime}$ are $\partial$-nonpositively homotopic; this means $\gamma(g)=\gamma\left(g^{\prime}\right)$ $=0$, thus $g \notin \mathscr{G}$, and hence $C \subset M$; therefore $M$ is open in $O^{n}-f\left(\partial I^{n}\right)$. Evidently $\bigcap g\left(\Pi^{n}\right)$ is closed in $O^{n}$, consequently $M$ is closed in $O^{n}-f\left(\partial \Pi^{n}\right)$.

(c) The assertion follows from (b).

For the application of our degree $\gamma$ we must provide sufficient conditions for $\gamma(F)=1$. In the remainder of this section we shall therefore be concerned with the problem of finding such conditions. To this end, we shall first introduce a degree $\tilde{\gamma}$ for a new class of maps.

Let $\Delta^{n-1}$ always be a euclidean $(n-1)$-polyhedron with $\partial \Delta^{n-1}=\varnothing$. In case $n=1$ let us make the following conventions:

$\partial \Delta^{0}=\varnothing$ means that $\Delta^{0}$ consists of an even number of points.

For any map $F: \Delta^{0} \rightarrow R_{*}, \gamma(F)$ is equal to zero or one according to whether the set $\left\{x \in \Delta^{0}: F(x)<0\right\}$ (and hence the set $\left\{x \in \Delta^{0}: F(x)>0\right\}$ ) consists of an even or an odd number of points. $\tilde{\gamma}(F)$.

We shall now assign to each continuous map $F: \Delta^{n-1} \rightarrow Q^{n+1}$ a degree

Choose $\delta>0$ such that $\max \left|F_{i}(x)\right|>2 \delta, \forall x \in \Delta^{n-1}$. For $\varepsilon \in(0, \delta)$ let $\sigma^{e}: \Delta^{n-1} \rightarrow R$ be defined by

$$
\sigma^{\varepsilon}(x):=\left\{\begin{array}{cl}
\varepsilon-\max _{i} F_{i}(x), & \text { if } \max _{i} F_{i}(x) \leqslant \varepsilon \\
-\varepsilon-\min _{i} F_{i}(x), & \text { if } \min _{i} F_{i}(x) \geqslant-\varepsilon \\
0, & \text { otherwise, }
\end{array}\right.
$$

and $F^{\varepsilon}: \Delta^{n-1} \rightarrow Q^{n+1}$ by $F^{\varepsilon}(x):=F(x)+\sigma^{\varepsilon}(x)$. Now we put:

$$
\tilde{\gamma}(F):=\gamma\left(\tilde{F}^{\varepsilon}\right), \quad \varepsilon \in(0, \delta),
$$

where $\widetilde{F}^{\varepsilon}$ stands for the map $\left(F_{1}^{\varepsilon}, \ldots, F_{n}^{\varepsilon}\right): \Delta^{n-1} \rightarrow R_{*}^{n}$.
From Theorem 4.1 it follows that $\tilde{\gamma}$ is well-defined and, moreover, invariant under homotopy, i.e.

LEMMA 4.4. Every homotopy $H: \Delta^{n-1} \times[0,1] \rightarrow Q^{n+1}$ satisfies $\tilde{\gamma}\left(H^{0}\right)$ $=\tilde{\gamma}\left(H^{1}\right)$.

LemMA 4.5. Let $F: \Delta^{n-1} \rightarrow Q^{n+1}$ be a continuous map and $K$ a simplicial decomposition of $\Delta^{n-1}$ such that there are labelings $\varphi: \hat{K} \rightarrow\{-1, \ldots,-(n+1)\}$, $\psi: \hat{K} \rightarrow\{1, \ldots, n+1\}$ satisfying $(5)$. Then $\tilde{\gamma}(F) \equiv \alpha_{\mathbb{K}}^{\varphi}(-1, \ldots,-n) \bmod 2$.

Proof. Let $\tilde{\varphi}$ be a labeling of $K$ generated by $\left(F_{1}, \ldots, F_{n}\right)$ such that $\tilde{\varphi}(x)>0$ if $\varphi(x)=-(n+1)$, and $\tilde{\varphi}(x)=\varphi(x)$ otherwise. Then $\tilde{\gamma}(F) \equiv$ $\equiv \alpha_{K}^{\bar{T}}(-1, \ldots,-n) \equiv \alpha_{K}^{\varphi}(-1, \ldots,-n) \bmod 2$.

The next theorem reveals the reason for introducing $\tilde{\gamma}$.

THEOREM 4.6. Let $F: \Pi^{n} \rightarrow R_{*}^{n+1}$ be continuous such that $F\left(\partial \Pi^{n}\right) \subset Q^{n+1}$ Suppose the restriction $F \mid \partial \Pi^{n}$ satisfies $\tilde{\gamma}\left(F \mid \partial \Pi^{n}\right)=1$. Then $\gamma(F) \neq \gamma(-F)$.

Proof. Choose $\varepsilon>0, \delta>0$ such that $\max \left|F_{i}(x)\right|>3 \varepsilon, \forall x \in \Pi^{n}$, and $d(F(x), F(y)) .<\varepsilon, \forall x, y \in \Pi^{n}$ such that $d(x, y) \leqslant \delta$. Define $\sigma^{\varepsilon}: \Pi^{n} \rightarrow R$ by $(7)$ Let $\tau: R \rightarrow[0,1]$ be a continuous map such that $\tau(0)=1$ and $\tau(x)=0, \forall x \geqslant \delta$. Define a homotopy $H: \Pi^{n} \times[0,1] \rightarrow R_{*}^{n+1}$ by $(x, t) \rightarrow F(x)+$ $+t \tau\left(d\left(x, \partial \Pi^{n}\right)\right) \sigma^{\varepsilon}(x)$. Then $H\left(\partial \Pi^{n} \times[0,1]\right) \subset Q^{n+1}, H^{0}=F$, and $H^{1},-H^{1}$ are $\partial$-negative. Therefore $F$ and $-F$ can be assumed to be $\partial$-negative without loss of generality. There exists a simplicial decomposition $K$ of $\Pi^{n}$ such that there are labelings $\varphi, \psi$ of $K$, generated by $F$ resp. by $-F$, such that

$$
\psi(x)=-\varphi(x), \quad \forall x \in \hat{K}-\delta K \text {. }
$$

Lemma 4.5 yields

$$
\alpha_{\zeta K}^{\varphi}(-1, \ldots,-n) \equiv 1 \bmod 2 .
$$

By Lemma 1.3 we obtain

$$
\alpha_{K}^{\varphi}(1, \ldots, n+1) \equiv \alpha_{K}^{\varphi}(-1, \ldots,-(n+1))+\alpha_{\delta_{K}}^{\varphi}(-1, \ldots,-n) \bmod 2 .
$$

From

and

$$
\alpha_{K}^{\varphi}(1, \ldots, n+1) \equiv \gamma(F) \bmod 2
$$

$$
\alpha_{k}^{\mu}(-1, \ldots,-(n+1))=\alpha_{k}^{\psi}(1, \ldots, n+1) \equiv \gamma(-F) \bmod 2
$$

the assertion follows.

Because of the foregoing theorem we now focus our attention to sufficient conditions for $\tilde{\gamma}(F)=1$.

At first we shall turn to the case $\Pi^{n}=\left\{x \in R^{n}:\|x\| \leqslant 1\right\}$; note that $\partial \Pi^{n}$ $=O^{n-1}$.

Theorem 4.7. Let $F: O^{n-1} \rightarrow Q^{n+1}$ be continuous. Suppose the map $\tilde{F}$ : $=\left(F_{1}, \ldots, F_{n}\right)$ of $O^{n-1}$ into $R^{n}$ satisfies at least one of the following conditions: 
(8) $\tilde{F}$ has no nonnegative or no nonpositive eigenvalue;

(9) There is no antipodal pair $x,-x$ and no $\lambda \in R_{+}$such that $\widetilde{F}(x)=\lambda \widetilde{F}(-x)$.

Then $\tilde{\gamma}(F)=1$.

Pro of. We shall prove $\gamma(\tilde{F})=1$. From this the assertion follows because of Theorem 4.1. If $\widetilde{F}(x)=-\widetilde{F}(-x), \forall x \in O^{n-1}$, then $\gamma(\widetilde{F})=\gamma(-\widetilde{F})=1$; this follows from well-known combinatorial results (see, for example, Fan [2] or Sperner [13]). So it suffices to show that, if $\tilde{F}$ satisfies (8) or (9), there exists a homotopy $H: O^{n-1} \times[0,1] \rightarrow R_{*}^{n}$ such that $H^{0}=\tilde{F}$ and $H^{1}(x)=-H^{1}(-x)$, $\forall x \in O^{n-1}$.

Case (8). We can assume that $\tilde{F}$ has no nonnegative eigenvalue; otherwise consider $-\widetilde{F}$. The desired homotopy can be defined by $H(x, t):=\widetilde{F}(x)-$ $-t(x+\tilde{F}(x))$.

Case (9). The homotopy $H$ can be defined by $H(x, t):=\tilde{F}(x)-\frac{1}{2} t(\tilde{F}(x)+$ $+\tilde{F}(-x))$

Most important with respect to applications is the case $\Pi^{n}=\Sigma^{n}$, which will now be studied in detail. We begin with some terminology.

A map $F: \partial \Sigma^{n} \rightarrow R^{n+1}$ is called Sperner-map (strong Sperner-map), if, for every $x \in \partial \Sigma^{n}$, at least one of the values $F_{i}(x), i \in I_{+}(x)$, is nonpositive (negative); $F$ is said to be a Fan-map, if, for every $x \in \partial \Sigma^{n}$, at least one of the values $F_{i}(x)$, $i \in I_{0}(x)$, is nonpositive. If the restriction $G \mid \partial \Sigma^{n}$ of a map $G: \Sigma^{n} \rightarrow R^{n+1}$ is a Sperner-map (Fan-map), then $G$ is called Sperner-map (Fan-map) too.

The main tool for the investigation of the case $\Pi^{n}=\Sigma^{n}$ will be Sperner's lemma. From this we obtain

LEMMA 4.8. If $F: \partial \Sigma^{n} \rightarrow Q^{n+1}$ is a continuous, positive, strong Sperner-map, then $\tilde{\gamma}(F)=1$.

Pr o of. There exist a simplicial subdivision $K$ of $\partial \Sigma^{n}$ and labelings $\varphi, \psi$ of $K$ that both satisfy (5) and are such that $-\varphi(x) \in I_{+}(x)$ and $\psi(x) \in\{1, \ldots, n+1\}$, $\forall x \in \hat{K}$. By Sperner's lemma we have $\alpha_{\mathrm{K}}^{\varphi}(-1, \ldots,-n) \equiv 1 \bmod 2$, and consequently by Lemma $4.5 \tilde{\gamma}(F)=1$.

Definition 4.9. A map $F: \partial \Sigma^{n} \rightarrow Q^{n+1}$ is called $S$-map, if there is a homotopy $H: \partial \Sigma^{n} \times[0,1] \rightarrow Q^{n+1}$ such that $H^{0}=F$ or $H^{0}=-F$ and such that $H^{1}$ is a positive, strong Sperner-map. A continuous map $F: \Sigma^{n} \rightarrow R_{*}^{n+1}$ is said to be an $S$-map, if $F \mid \partial \Sigma^{n}$ is an $S$-map.

From Theorem 4.6, Lemma 4.8 and Lemma 4.4 we derive

THEOREM 4.10. If $F: \Sigma^{n} \rightarrow R_{*}^{n+1}$ is an S-map, then $\gamma(F) \neq \gamma(-F)$.

In the next theorem we present several conditions which are sufficient for a map to be an $S$-map.

THEOREM 4.11. If a continuous map $F: \partial \Sigma^{n} \rightarrow Q^{n+1}$ satisfies at least one of the following conditions, then $F$ is an S-map:
(10) $F$ is a Sperner-map, and there is no $x \in \partial \Sigma^{n}$ such that $F(x) \leqslant 0$ and $x F(x)$ $=0$;

(11) There is a continuous map $G: \partial \Sigma^{n} \rightarrow R^{n+1}$ such that $F(x)+t G(x) \in Q^{n+1}$, $\forall x \in \partial \Sigma^{n}, \forall t \in[0,1]$, and such that $F+G$ satisfies (10);

(12) $F$ is a Fan-map without any negative eigenvalue;

(13) There are no $x \in \partial \Sigma^{n}, \lambda \in R_{+}$such that $F(x) \leqslant \lambda x$ and $F_{i}(x)=\lambda x_{i}$, $\forall i \in I_{+}(x)$;

(14) There is $a \in I\left(\Sigma^{n}\right)$ such that there are no $x \in \partial \Sigma^{n}, \lambda \in R_{+}$with $F(x) \leqslant \lambda a$ and $F_{i}(x)=\lambda a_{i}, \forall i \in I_{+}(x)$

(15) There is no $x \in \partial \Sigma^{n}$ such that $F_{i}(x)=0, \forall i \in I_{+}(x)$, or such that $F_{j}(x)>0$ for a $j \in I_{+}(x), F_{i}(x)=0, \forall i \in I_{+}(x)-\{j\}$ and $F_{k}(x) \leqslant 0, \forall k$ with $k<j$;

(16) There is no $x \in \partial \Sigma^{n}$ such that $F_{i}(x) \geqslant 0, \forall i \in I_{+}(x), F_{i}(x)=0, \forall i \in I_{+}(x)$ with $x_{i}<\max x_{j}$, and $F_{i}(x) \leqslant 0, \forall i \in I_{0}(x)$;

(17) There is a $a \in\left(\Sigma^{n}\right)$ such that there is no $x \in \partial \Sigma^{n}$ with $F_{i}(x) \geqslant 0, \forall i \in I_{+}(x)$, $F_{i}(x)=0, \forall i \in I_{+}(x)$ with $x_{i}<a_{i}$, and $F_{i}(x) \leqslant 0, \forall i \in I_{0}(x)$;

(18) There are no $x \in \partial \Sigma^{n}, \lambda, \mu \in R_{+}$such that $F(x)+\lambda=\mu x$;

(19) There are no $x \in \partial \Sigma^{n}, \lambda \in R_{+}$such that $F(x)+\lambda \geqslant 0$ and $F_{i}(x)+\lambda=0, \forall i$ with $x_{i}<\max _{j} x_{j}$.

Proof. Case (10). Choose $\varepsilon>0$ such that for every $x \in \partial \Sigma^{n}$ there is $i \in\{1, \ldots, n+1\}$ with $x_{i}>0$ and $F_{i}(x)<-2 \varepsilon$ or with $F_{i}(x)>2 \varepsilon$. Then define a continuous map $\sigma: \partial \Sigma^{n} \rightarrow R$ by

$$
\sigma(x):= \begin{cases}-\varepsilon, & \text { if } \max _{i} F_{i}(x) \geqslant 2 \varepsilon, \\ \varepsilon-\max _{i}\left\{F_{i}(x), 0\right\}, & \text { otherwise. }\end{cases}
$$

Now consider the homotopy $H: \partial \Sigma^{n} \times[0,1] \rightarrow Q^{n+1}$ carrying $(x, t)$ into $F(x)+$ $+t \sigma(x)$. Then $H^{0}=F$, and $H^{1}$ is a positive, strong Sperner-map.

Case (11) is reduced to case (10) by considering the homotopy $H$ : $\partial \Sigma^{n} \times$ $\times[0,1] \rightarrow Q^{n+1}$ defined by $(x, t) \rightarrow F(x)+t G(x)$.

Cases (12)-(17) follow from case (11) by putting in cases (12), (13)

$$
G(x):=-\min \left\{s \in R_{+}: x(F(x)-s x) \leqslant 0\right\} x,
$$

in case (14)

$$
G(x):=-\min \left\{s \in R_{+}: x(F(x)-s a) \leqslant 0\right\} a
$$

in case (15)

$$
G(x):=-\min \left\{s \in R_{+}: x(F(x)-s E(x)) \leqslant 0\right\} E(x),
$$


where $E: \partial \Sigma^{n} \rightarrow R^{n+1}$ is defined by $E_{i}(x):=i \max \left\{0, F_{i}(x)\right\}, i=1, \ldots, n+1$, in case (16)

$$
G_{i}(x):=-\frac{x_{i}}{\max _{1 \leqslant j \leqslant n+1} x_{j}} \max \left\{F_{i}(x), 0\right\}, i=1, \ldots, n+1,
$$

in case (17)

$$
G_{i}(x):=-\min \left\{\frac{x_{i}}{a_{i}}, 1\right\} \max \left\{F_{i}(x), 0\right\}, i=1, \ldots, n+1 .
$$

Cases (18), (19). Define a homotopy $H: \partial \Sigma^{n} \times[0,1] \rightarrow Q^{n+1}$ by $(x, t)$ $\rightarrow F(x)+t \max \left\{s \in R: F(x)+s \in Q^{n+1}\right\} ;$ then $H^{0}=F$, and $H^{1}$ satisfies (13) resp. (16).

§. Some topological consequences. Let $F: P^{n} \rightarrow R_{*}^{n+1}$ be continuous and $\partial$ nonpositive, and let $f: P^{n} \rightarrow O^{n}$ be defined by $f(x):=\frac{F(x)}{\|F(x)\|}, \forall x \in P^{n}$. Then $F$ is said to be ppositive-covering, if $f\left(I\left(P^{n}\right)\right)$ is a neighborhood of $\Sigma^{n}-f\left(\partial P^{n}\right)$ in $O^{n}$ (this implies $\Sigma^{n} \subset f\left(P^{n}\right)$ ).

By Theorem 4.3, each continuous $\partial$-nonpositive map $F$ of $\Pi^{n}$ into $R_{*}^{n+1}$ with $\gamma(F)=1$ is positive-covering.

For every $S$-map $F: \Sigma^{n} \rightarrow R_{*}^{n+1}$ let $\mathscr{H}(F)$ denote the class of maps $G: \Sigma^{n}$ $\rightarrow R_{*}^{n+1}$ such that $F$ and $G$ are $\partial$-nonpositively homotopic.

In the next theorem we shall refer to the following sentence:

$\mathscr{A}(F)$. If $G: \Sigma^{n} \rightarrow R^{n+1}$ is a continuous map such that $F$ and $G$ are together $\partial$-nonpositive and if $\varrho \in R_{+}$is arbitrary, then $\varrho F+G$ is positive-covering and has a positive eigenvalue, or $(F, G)$ has an equilibrium value $\leqslant-\varrho$.

THEOREM 5.1. Let $E: \Sigma^{n} \rightarrow R_{*}^{n+1}$ be an $S$-map such that there is $\tilde{F} \in \mathscr{H}(-E)$ violating $\mathscr{A}(\widetilde{F})$. Then $\mathscr{A}(F)$ holds for every map $F \in \mathscr{H}(E)$; in particular, all maps in $^{\circ} \mathscr{H}(E)$ are positive-covering and have a positive eigenvalue.

Proof. The assertion follows from Theorems 4.1, 4.2, 4.3, 4.10.

Essential for the application of Theorem 5.1 is the knowledge of sufficient conditions under which two $\partial$-nonpositive maps $F, G: \Sigma^{n} \rightarrow R^{n+1}$ are together $\partial$-nonpositive. We present here two such conditions each of which is immediately seen to be sufficient:

(20) For every $x \in \partial \Sigma^{n}$ there is $\lambda \in R_{+}$such that $\lambda F(x) \geqslant G(x)$.

(21) $F$ and $G$ are Fan-maps.

Notice that two Sperner-maps $F, G: \Sigma^{n} \rightarrow R^{n+1}$ are not necessarily together $\partial$-nonpositive.

Using condition (21) we can derive from Theorem 5.1:

THEOREM 5.2. Let $F: \Sigma^{n} \rightarrow R_{*}^{n+1}$ be continuous. Suppose there are $\varepsilon_{1}, \ldots, \varepsilon_{n+1}$ such that $\left\{\varepsilon_{i}: i=1, \ldots, n+1\right\}=\{-1,1\}$ and such that $F^{\prime}:=$ $\left(\varepsilon_{1} F_{1}, \ldots, \varepsilon_{n+1} F_{n+1}\right)$ is a Fan-map satisfying at least one of the following two conditions:

(22) $F^{\prime}$ has no negative eigenvalue;

(23) $-F^{\prime}$ is $\partial$-nonpositive and not positive-covering, and $F^{\prime} \mid \partial \Sigma^{n}$ has no negative eigenvalue.

Then $F$ has a positive and a negative eigenvalue.

Proof. Suppose $F$ satisfies the conditions of the theorem with certain $\varepsilon_{i}$. Define $G: \Sigma^{n} \rightarrow R_{*}^{n+1}$ by $x \rightarrow\left(\varepsilon_{1} x_{1}, \ldots, \varepsilon_{n+1} x_{n+1}\right)$. By Theorem $5.1,\left(\left(F^{\prime}, G\right)\right.$ and $\left(F^{\prime},-G\right)$, and hence $(F, i)$ and $(F,-i)\left(i: \Sigma^{n} \rightarrow R^{n+1}\right.$ the inclusion map) have negative equilibrium values.

Observe that Theorem 5.1 includes Brouwer's fixed point theorem: If $f: \Sigma^{n}$ $\rightarrow \Sigma^{n}$ is any continuous map, take $F:=i$ (inclusion map), $G:=-f, \varrho:=0$ and apply Theorem 5.1; hence there are $x \in \Sigma^{n}, \lambda \leqslant 0$ such that $-f(x)=\lambda x$, and as $f(x) \in \Sigma^{n}$ implies $\lambda=-1, x$ is a fixed point of $f$.

Moreover, Theorem 5.1 generalizes Corollary 2 of [4] and a well-known result (see [3, Theorem 3]) of G. Frobenius on real $n \times n$-matrices with nonnegative elements.

The next theorem also extends Brouwer's fixed point theorem and the mentioned result of Frobenius. The proof will heavily rely on Sperner's lemma

THEOREM 5.3. Let $F$ be a continuous map of a closed $n$-cell $C^{n}$ into $R_{*}^{n+1}$ such that $F(x) \neq \lambda F(y)$ for all distinct $x, y \in C^{n}$ and all $\lambda \in R_{+}$. Suppose there is $c \in C^{n}$ such that $F(c)>0$. If $H: C^{n} \times[0,1] \rightarrow R_{*}^{n+1}$ is any 0 -nonpositive homotopy such that $H^{0}=F$, then $H^{1}$ is positive-covering.

Proof. Without loss of generality we can assume that $H$ is a $\partial$-negative homotopy.

Let $f: C^{n} \rightarrow O^{n}$ be defined by $f(x):=\frac{F(x)}{\|F(x)\|}, \forall x \in C^{n}$. According to the assumption of the theorem, $f$ is injective, hence $f: C^{n} \rightarrow f\left(C^{n}\right)$ is topological. Therefore we may assume $C^{n} \subset O^{n}$ and $f=$ inclusion map. By assumption we have $C^{n} \cap \Sigma^{n} \neq \varnothing$, hence by Lemma 1.1 $\Sigma^{n} \subset I\left(C^{n}\right)$. For $i \in N$ let $K_{i}$ denote the $i$ th barycentric subdivision of the natural simplicial decomposition of $O^{n}$. To each complex $K_{i}$ we associate an $n$-dimensional subcomplex $L_{i}$ consisting of the faces of all $n$-simplexes $\sigma^{n} \in K_{i}$ such that $\sigma^{n} \subset C^{n}$. Let $\Pi_{i}:=\left\langle L_{i}\right\rangle$, and let $F^{i}, f^{i}$, $H^{1, i}$ be the restrictions of $F$ resp. $f$ resp. $H^{1}$ to $\Pi_{i}, \forall i \in N$. Since $H$ is a $\partial$-negative homotopy, there is $i_{0} \in N$ such that $\Sigma^{n} \cap H\left(\partial \Pi_{i} \times[0,1]\right)=\varnothing, \forall i>i_{0}$. If $i>i_{0}$, then, for every $x \in \hat{L}_{i} \cap \partial \Sigma^{\prime \prime}$ and every labeling $\varphi$ of $L_{i}$ generated by $f^{i}$, we have $\varphi(x) \in I_{+}(x)$; from Sperner's lemma we infer $\gamma\left(F^{i}\right)=\gamma\left(f^{i}\right) \equiv \alpha_{L_{i}}^{\varphi}(1, \ldots, n+1)$ $\equiv 1 \bmod 2$, hence $\gamma\left(H^{1, i}\right)=1, \forall i>i_{0}$. Application of Theorem 4.3 completes our proof.

In the next part of this section we shall use our degree $\gamma$ as a tool for establishing some theorems for set-válued mappings, which extend the classical 
fixed point theorem of Kakutani [8]. We start with some terminology.

For every subset $A$ of $R^{n}$ let $\mathscr{K}(A)$ denote the set of all nonempty compact convex subsets of $A$. For any maps $\Phi, \Psi$ of $\Sigma^{n}$ into $\mathscr{K}\left(R^{n+1}\right)$ let $\lambda \Phi(\lambda \in R)$ and $\Phi+\Psi$ be defined by $x \rightarrow \lambda \Phi(x)$ resp. $x \rightarrow \Phi(x)+\Psi(x)$; obviously $\lambda \Phi$ and $\Phi+\Psi$ are maps of $\Sigma^{n}$ into $\mathscr{K}\left(R^{n+1}\right)$

$\Phi$ is called upper semicontinuous, if $\lim x^{i}=x^{0}\left(x^{i} \in \Sigma^{n}, i \in N\right), y^{i} \in \Phi\left(x^{i}\right)$, $\lim y^{i}=y^{0}$ always imply $y^{0} \in \Phi\left(x^{0}\right)$;

$\Phi$ is called bounded, if $\bigcup \Phi(x)$ is bounded;

$\Phi$ is called $\partial$-positive, if the set $\Phi(x) \cap-R_{+}^{n+1}$ is empty for every $x \in \partial \Sigma^{n}$; $\Phi$ is called Fan-map, if for every $x \in \partial \Sigma^{n}$ there is $y \in \Phi(x)$ such that $y_{i} \leqslant 0$, $\forall i \in I_{0}(x)$;

$\Phi$ is called positive-covering, if for every $x \in R_{+}^{n+1}$ there are $\lambda \in R_{+}$and $y \in \bigcup$

if there are $x \in \Sigma^{n}, y \in \Phi(x), z \in \Psi(x), \lambda \in R$ such that $\lambda y=z$, then $\lambda$ is called equilibrium value of $(\Phi, \Psi)$; in the special case $\Phi(x)=\{x\}, \forall x \in \Sigma^{n}, \lambda$ is called eigenvalue of $\Psi$

Throughout the remaining part of this paper it will tacitly be understood that all set-valued maps $\Phi, \Psi$ are bounded and upper semicontinuous.

Essential for our next theorems are the following two sentences:

$\mathscr{A}(\Phi, \Psi)$. If $\varrho \in R_{+}$is arbitrary, then $\varrho \Phi+\Psi$ is positive-covering and has a positive eigenvalue, or $(\Phi, \Psi)$ has an equilibrium value $\leqslant-\varrho$.

$\mathscr{B}(\Phi) . \mathscr{A}(\Phi, \Psi)$ holds for every map $\Psi: \Sigma^{n} \rightarrow \mathscr{K}^{\prime}\left(R^{n+1}\right)$ such that $\Psi(x) \cap-R_{+}^{n+1} \neq \varnothing, \forall x \in \partial \Sigma^{n}$.

THEOREM 5.4. Let $\Phi: \Sigma^{n} \rightarrow \mathscr{K}\left(\boldsymbol{R}_{*}^{n+1}\right)$ be a j-positive Fan-map. Then $\mathscr{A}(\Phi, \Psi)$ holds for every Fan-map $\Psi: \Sigma^{n} \rightarrow \mathscr{K}\left(R^{n+1}\right)$, or $\mathscr{B}(-\Phi)$ holds.

- Proof. Our proof will be much along the lines of Kakutani's proof of his fixed point theorem; his way of reasoning in [8] will here be assumed as known.

Let $K_{i}$ be the $i$ th barycentric subdivision of $\Sigma^{n}(i \in N)$. For every $i \in N$ we assign to each vertex $x \in \widehat{K}_{i}$ a $y \in \Phi(x)$ in such a way that the linear extension of this map inside each simplex of $K_{i}$ yields a continuous Fan-map $F^{i}: \Sigma^{n} \rightarrow R^{n+1}$ in the sense of our definition in $\S 4$. Let $i_{0} \in N$ be such that, for all $i>i_{0}$, $0 \notin F^{i}\left(\Sigma^{n}\right)$ holds and $-F^{i}$ is $\partial$-negative. From Theorems 4.10 and 4.11 we infer $\gamma\left(F^{i}\right) \neq \gamma\left(-F^{i}\right), \forall i>i_{0}$. Suppose $\mid\left\{i \in N: i>i_{0}\right.$ and $\left.\gamma\left(F^{i}\right)=1\right\} \mid=\infty$; to each Fan-map $\Psi: \Sigma^{n} \rightarrow \mathscr{K}\left(R^{n+1}\right)$ we can associate, for every $i \in N$, a Fan-map $G^{l}: \Sigma^{n}$ $\rightarrow R^{n+1}$ in the way described above; application of Theorem 5.1 and passing to the limit yield $\mathscr{A}(\Phi, \Psi)$. Similarly we conclude that $\mid\left\{i \in N: i>i_{0}\right.$ and $\gamma\left(-F^{i}\right)$ $=1\} \mid=\infty$ implies $\mathscr{B}(-\Phi)$.

We obtain Kakutani's theorem from Theorem 5.4 by taking $\Phi(x):=\{x\}$, $\forall x \in \Sigma^{n}$; then $-\Phi$ is not positive-covering, and therefore, for each given map $\Psi: \Sigma^{n} \rightarrow \mathscr{K}\left(-\Sigma^{n}\right)$, there is $x \in \Sigma^{n}$ such that $x \in-\Psi(x)$

The proofs of the next two results will not be given here, because they are analegous to the proof of Theorem 5.4.
Trerem 5.5. Let $\Phi: \Sigma^{n} \rightarrow \mathscr{K}^{\prime}\left(R_{*}^{n+1}\right)$ be $\partial$-positive and such that, for each $x \in \cap \Sigma^{n}$, there is $y \in \Phi(x)$ with $x y \leqslant 0$. Then $\mathscr{B}(\Phi)$ or $\mathscr{B}(-\Phi)$.

Theorem 5.5 includes a result of D. Gale [7, Principal Lemma].

THForem 5.6. Let $\Phi, \Psi: \Sigma^{n} \rightarrow \mathscr{H}^{\prime}\left(R^{n+1}\right)$ be Fan-maps; suppose $\Phi$ has no nonpositive eigenvalue. Then $\mathscr{\mathcal { A }}(\Phi, \Psi)$.

As an easy consequence of Theorem 5.6 we receive

Corol.tary 5.7. Every Fan-map $\Phi: \Sigma^{n} \rightarrow \mathscr{K}\left(R^{n+1}\right)$ has an eigenvalue.

Now we turn to a fundamental technique developed by Fan (see, for example, [6]), which extends the covering theorem of Knaster-KuratowskiMazurkiewicz to the infinite-dimensional case and has been used by Fan and Mazurkiewicz to the inthematicians, for example H. Brézis, L. Nirenberg, G. Stampacchia [1], other mathematicians, for example H. Brezis, L. Nirenberg, G. Stampace

deriving maser, $V$ denotes a real Hausdorf Throughout the remace. Note that every $n$-dimensional subspace of $V$ is

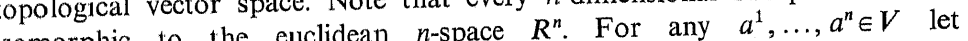
isomorphic to the euch of $a^{1}, \ldots, a^{n}$.

nv $\left(a^{1}, \ldots, a^{n}\right)$ denote the convex hull of $a^{1}, \ldots, a^{n}$.

Here we shall establish a theorem which should be compared with Theorem 1 of Brézis-Nirenberg-Stampacchia $[1]$, in $[1]$ both

Let $K$ be a convex subset of $V$, and let $\varphi: K \times K \rightarrow R$ be a map The properties:

(24) For every fixed $y \in K, \varphi(x, y)$ is a continuous function of $x$ on the intersection of $K$ with any finite-dimensional subspace of $V$

(25) The set $\{x \in K: \varphi(x, y)=0\}$ is closed in $K$ for all $y \in K$ and compact for at least one $y$;

(26) $\varphi(x, x) \leqslant 0, \forall x \in K$

(27) For every $x \in K$, the set $\{y \in K: \varphi(x, y)>0\}$ is convex;

(28) For every $x \in K$, at least one of the sets $\{y \in K: \varphi(x, y) \geqslant 0\}$, $\{y \in K: \varphi(x, y)=0\}$ is convex;

(29) For every $x \in K, \varphi(x, x)=0$ implies $\varphi(x, y) \geqslant 0, \forall y \in K$;

For every finite subset $X$ of $K$ there is a compact convex subset $K_{0}$, $X \subset K=K$ with the following property: For every $z \in K_{0}$ there is $X \subset K_{0} \subset K$, with ${ }^{2}$ of $z$ in $K_{0}$ and $a y \in K_{0}$ such that $\varphi(x, y) \geqslant 0$, $\forall x \in N(z)$.

Then there is $x^{0} \in K$ such that $\varphi\left(x^{0}, y\right)=0, \forall y \in K$.

Proof. If $K$ contains only one vector, the assertion is trivial. Therefore we Pre an infinite set. If we associate to every $y \in K$ the set $S(y):=\{x \in K: \varphi(x, y)=0\}$, then we can prove the assertion $\bigcap S(y) \neq \varnothing$ in the following way: Because of the compactness of at least one $\stackrel{y \in K}{(y)}$, it is enough 
to show that, for any given finite subset $X$ of $K$, the intersection $\bigcap_{x \in X} S(x)$ is nonempty. Choose $x^{\prime} \in K-X$ and put $X^{\prime}:=X \cup\left\{x^{\prime}\right\}$. For $X^{\prime}$ there exists a compact convex subset $K_{0}$ of $K$ with the property mentioned in (30). Because of the compactness of $K_{0}$, there exists a finite set $Y=\left\{y^{1}, \ldots, y^{m}\right\}, X \subset Y \subset K_{0}$, with the following property:

(31) For every $x \in K_{0}$ there is $y \in Y$ such that $\varphi(x, y) \geqslant 0$.

Now consider the continuous maps $F: \operatorname{conv}\left(y^{1}, \ldots, y^{m}\right) \rightarrow R^{m}$ and $T: \Sigma^{m-1}$ $\rightarrow \operatorname{conv}\left(y^{1}, \ldots, y^{m}\right)$, defined by $F_{i}(x):=\varphi\left(x, y^{i}\right), \quad i=1, \ldots, m$, resp. $T(x):=x_{1} y^{1}+\ldots+x_{m} y^{m}$. By Theorem 5.1, it suffices to show that the composed map $F \circ T$. $\Sigma^{m-1} \rightarrow R^{m}$ has a zero or is an $S$-map. We shall do so by applying Theorem 4.11 with condition (10). Because of $\operatorname{conv}\left(y^{1}, \ldots, y^{m}\right) \subset K_{0}$ and (31), we have $(F \circ T)(x) \nless 0, \forall x \in \partial \Sigma^{m-1}$. From (26), (27) we infer $\operatorname{conv}\left(a^{1}, \ldots, a^{n}\right) \subset \bigcup_{i=1}^{n}\left\{x \in K: \varphi\left(x, a^{i}\right) \leqslant 0\right\}$ for all $a^{1}, \ldots, a^{n} \in K$; this means that $F \circ T$ is a Sperner-map. Let $x$ be any given vector of $\partial \Sigma^{m-1}$ such that $\left(F_{i} \circ T\right)(x)=0, \forall i \in I_{+}(x)$. Then $\varphi\left(T(x), y^{i}\right)=0, \forall i \in I_{+}(x)$. From (28) it follows $\varphi(T(x), T(x))=0$. (29) yields $\varphi\left(T(x), y^{i}\right) \geqslant 0$, i.e. $F_{i}(T(x)) \geqslant 0$ for $i$ $=1, \ldots, m$, and hence $(F \circ T)(x) \geqslant 0$.

The following result, which is an easy consequence of the preceding theorem, should be compared with Application 1 in [1].

COROLlary 5.9. Let $K$ be a nonempty compact convex subset of $V$, and let $\varphi$ : $K \times K \rightarrow R$ be a map such that (26), (29) are satisfied, and, for every fixed $x \in K$, $\varphi(x, y)$ is a concave function of $y$ on $K$, and, for every fixed $y \in K, \varphi(x, y)$ is a continuous function of $x$ on $K$. Suppose that, for every $z \in K$, there is a neighborhood $N(z)$ of $z$ in $K$ and $a y \in K$ such that $\varphi(x, y) \geqslant 0, \forall x \in N(z)$. Then there is $x^{0} \in K$ such that $\varphi\left(x^{0}, y\right)=0, \forall y \in K$.

\section{References}

[1] H. Brézis, L. Nirenberg and G. St a mpacchia, A remark on Ky Fan's minimax principle, Boll. Un. Mat. Ital. 6 (1972), pp. 293-300.

[2] K y F an, A generalization of Tucker's combinatorial lemma with topological applications, Ann. of Math. 56 (1952), pp. 431-437.

[3] - Topological proofs for certain theorems on matrices with non-negative elements, Monatshefte f. Math. 62 (1958), pp. 219-237.

[4] - A covering property of simplexes, Math. Scand. 22 (1968), pp. 17-20.

[5] - A combinatorial property of pseudomanifolds and covering properties of simplexes, J. Math. Anal. and Appl. 31 (1970), pp. 68-80.

[6] - A minimax inequality and applications, In: Inequalities III (O. Shisha, ed.), Academic Press, New York-London 1972, pp. 103-113.

[7] D. Gale, The law of supply and demand, Math. Scand. 3 (1955), pp. 155-169.

[8] S. Kakut a ni, A generalization of Brouwer's fixed point theorem, Duke Math. J. 8 (1941), pp.
457-459.
[9] B. Knaster, C. Kuratowski und S. Mazurkiewicz, Ein Beweis des Fixpunktsatzes fur ndimènsionale Simplexe, Fund. Math. 14 (1929), pp. 132-137.

[10] M. A. Kras nosel's kii, Topological Methods in the Theory of Nonlinear Integral Equations, Pergamon Press, New York 1964.

[11] H. Sies, Abbildungsgrad und Spernersches Lemma, Doctoral Dissertation, Universitat Hamburg 1979.

[12] E. S perner, Neuer Beweis fur die Invarianz der Dimensionszahl und des Gebietes, Abh. Math. Sem. Univ. Hamburg 6 (1928), pp. 265-272.

[13] - Kombinatorik bewerteter Komplexe, Abh. Math. Sem. Univ. Hamburg 39 (1973), pp. 21-43.

Accepté par la Rédaction le 16.3.1981 